TRANSACTIONS OF THE

AMERICAN MATHEMATICAL SOCIETY

Volume 355, Number 7, Pages 2813-2828

S 0002-9947(03)03282-3

Article electronically published on March 12, 2003

\title{
GALOIS GROUPS OF QUANTUM GROUP ACTIONS AND REGULARITY OF FIXED-POINT ALGEBRAS
}

\author{
TAKEHIKO YAMANOUCHI
}

Dedicated to Professor Masamichi Takesaki on the occasion of his seventieth birthday

\begin{abstract}
It is shown that, for a minimal and integrable action of a locally compact quantum group on a factor, the group of automorphisms of the factor leaving the fixed-point algebra pointwise invariant is identified with the intrinsic group of the dual quantum group. It is proven also that, for such an action, the regularity of the fixed-point algebra is equivalent to the cocommutativity of the quantum group.
\end{abstract}

\section{INTRODUCTION}

When given an action $\alpha$ of a locally compact quantum group $\mathbb{G}$ on a von Neumann algebra $A$, one may associate to it the subgroup Aut $\left(A / A^{\alpha}\right)$ of all automorphisms of $A$ leaving the fixed-point algebra $A^{\alpha}$ invariant pointwise. Let us call this subgroup "the Galois group of $\alpha$ ". As [1, Theorem III.3.3] suggests, it would sometimes happen (or be expected) that the Galois group carries an important piece of information on the quantum group $\mathbb{G}$ itself. With this philosophy in mind, we started in [17] to investigate Galois groups of minimal actions of compact Kac algebras on factors by making good use of the Galois correspondence established by Izumi, Longo and Popa [7]. In [20], we succeeded in describing the Galois group of any minimal action of a compact Kac algebra as the so-called intrinsic group of the dual discrete Kac algebra. This extended the result of [1] cited above. As an application, we were able to show that, if the quantum group in question is finite-dimensional, then its cocommutativity is equivalent to the regularity of the fixed-point algebra. Our main goal of this paper is to extend these results to a larger class of locally compact quantum groups, not only compact Kac algebras. If we try to achieve this goal exactly along the line carried out in [17] and [20], then a Galois correspondence for a (minimal) action of a more general locally compact quantum group would certainly be needed. At the moment, it seems that the results of Enock in [4] would answer this purpose. Unfortunately, there are however a few mistakes in his proofs, and, to the best of the author's knowledge, they have not been restored yet. So we cannot apply Enock's Galois correspondence to the situation we will consider in this paper. Therefore, we will adopt a new approach here that does not resort to any Galois correspondence.

Received by the editors June 24, 2002 and, in revised form, November 6, 2002.

2000 Mathematics Subject Classification. Primary 46L65; Secondary 22D25, 46L10, 81R50.

Key words and phrases. Locally compact quantum group, action, factor, regularity. 
The outline of this paper is the following. In Section 1, we fix the notation used in the whole of our discussion. Basic facts about locally compact quantum groups (in the sense of Kustermans and Vaes) and their actions on von Neumann algebras are collected. In Section 2, we will prove that the Galois group of a minimal, integrable action of a locally compact quantum group $\mathbb{G}$ is topologically isomorphic to the intrinsic group of the dual $\widehat{\mathbb{G}}$. Section 3 is concerned with regularity of the fixed-point algebra of a minimal, integrable action. We prove that the regularity considerably determines the structure of the quantum group. Namely it is shown, with some exception, that the fixed-point algebra is regular if and only if the locally compact quantum group under consideration is cocommutative. In Section 4, we make a few remarks on the Izumi-Longo-Popa Galois correspondence. One of them concerns an explicit formula for the inverse map of their Galois correspondence. Finally, we include an Appendix for some auxiliary results which are applied to the argument made in Section 3.

The author is grateful to Professors Michel Enock and Stefaan Vaes for having informed him that there are mistakes in some proofs in [4]. He is also indebted to Professor Masaki Izumi for indicating an error in the earlier draft of the manuscript.

\section{TERminology AND NOTATION}

Given a von Neumann algebra $A$ and a faithful normal semifinite weight $\phi$ on $A$, we introduce the subsets $\mathfrak{n}_{\phi}, \mathfrak{m}_{\phi}$ and $\mathfrak{m}_{\phi}^{+}$of $A$ by

$$
\mathfrak{n}_{\phi}=\left\{x \in A: \phi\left(x^{*} x\right)<\infty\right\}, \quad \mathfrak{m}_{\phi}=\mathfrak{n}_{\phi}^{*} \mathfrak{n}_{\phi}, \quad \mathfrak{m}_{\phi}^{+}=\mathfrak{m}_{\phi} \cap A_{+} .
$$

The standard (GNS) Hilbert space obtained from $\phi$ is denoted by $H_{\phi}$. We use the symbol $\Lambda_{\phi}$ for the canonical embedding of $\mathfrak{n}_{\phi}$ into $H_{\phi}$. The modular objects such as the modular operator, the modular conjugation, the $S$-operator, the $F$ operator, the modular automorphism group, etc. associated to $\phi$ are denoted by $\nabla_{\phi}, J_{\phi}, S_{\phi}, F_{\phi}, \sigma^{\phi}$, respectively. (Since we follow the notation employed in [10], the symbol $\nabla$ will be used to denote the modular operator of a weight.) The set of unitaries in $A$ is denoted by $\mathcal{U}(A)$. For a von Neumann subalgebra $B$ of $A$, define $\mathcal{N}(B):=\left\{u \in \mathcal{U}(A): u B u^{*}=B\right\}$ and call it the normalizer (group) of $B$ in $A$.

We let $B(H)$ stand for the algebra of all bounded operators on a Hilbert space $H$.

\subsection{Locally compact quantum groups.}

Definition 2.1. Following [10] (see [9] also), we say that a quadruple $\mathbb{G}=(M$, $\Delta, \varphi, \psi$ ) is a locally compact quantum group (in the von Neumann algebra setting) or a von Neumann algebraic quantum group if

(1) $M$ is a von Neumann algebra;

(2) $\Delta$ is a unital normal injective $*$-homomorphism from $M$ into $M \otimes M$ satisfying $(\Delta \otimes i d) \circ \Delta=(i d \otimes \Delta) \circ \Delta$

(3) $\varphi$ is a faithful normal semifinite weight on $M$ such that

$$
\varphi((\omega \otimes i d)(\Delta(x)))=\varphi(x) \omega(1) \quad\left(\forall \omega \in M_{*}^{+}, \forall x \in \mathfrak{m}_{\varphi}^{+}\right) ;
$$

(4) $\psi$ is a faithful normal semifinite weight on $M$ such that

$$
\psi((i d \otimes \omega)(\Delta(x)))=\psi(x) \omega(1) \quad\left(\forall \omega \in M_{*}^{+}, \forall x \in \mathfrak{m}_{\psi}^{+}\right) .
$$


Let us fix a locally compact quantum group $\mathbb{G}=(M, \Delta, \varphi, \psi)$ throughout the rest of this section. We will always think of $M$ as represented on the GNS-Hilbert space $H_{\varphi}$ obtained from $\varphi$. By the left invariance of $\varphi$, one gets a unitary $W(\mathbb{G})$ on $H_{\varphi} \otimes H_{\varphi}$ characterized by

$$
W(\mathbb{G})^{*}\left(\Lambda_{\varphi}(x) \otimes \Lambda_{\varphi}(y)\right)=\Lambda_{\varphi \otimes \varphi}(\Delta(y)(x \otimes 1)) \quad\left(x, y \in \mathfrak{n}_{\varphi}\right) .
$$

This unitary is called the Kac-Takesaki operator of $\mathbb{G}$, and is denoted simply by $W$ if there is no danger of confusion. The modular operator and the modular conjugation of $\varphi$ will be denoted simply by $\nabla$ and $J$. The unitary antipode, the scaling group and the scaling constant of $\mathbb{G}$ are respectively denoted by $R,\left\{\tau_{t}\right\}_{t \in \mathbf{R}}$, $\nu(>0)$. As in [10, we assume that $\psi=\varphi \circ R$.

According to [10, there canonically exists another locally compact quantum group $\widehat{\mathbb{G}}=(\widehat{M}, \hat{\Delta}, \hat{\varphi}, \hat{\psi})$, called the locally compact quantum group dual to $\mathbb{G}$ such that $\left\{\widehat{M}, H_{\varphi}\right\}$ is a standard representation. So we always regard $\widehat{M}$ as acting on $H_{\varphi}$. In fact, $\widehat{M}$ is by definition the von Neumann algebra generated by $\{(\omega \otimes i d)(W)$ : $\left.\omega \in M_{*}\right\}$. The mapping $\lambda: \omega \in M_{*} \longmapsto(\omega \otimes i d)(W) \in \widehat{M}$ is called the left regular representation of $\mathbb{G}$. There is a canonical identification (= the Fourier transform) of $H_{\hat{\varphi}}$ with $H_{\varphi}$. So we consider the modular operator and the modular conjugation of $\hat{\varphi}$, denoted by $\hat{\nabla}$ and $\hat{J}$, as acting on $H_{\varphi}$. The unitary antipode, the scaling group and the modular element of $\widehat{\mathbb{G}}$ are denoted respectively by $\hat{R}, \hat{\tau}, \hat{\delta}$. We say that $\mathbb{G}$ is compact if $\varphi(1)<\infty$. In this case, we agree to take $\varphi$ to be a state. We say that $\mathbb{G}$ is discrete if $\widehat{\mathbb{G}}$ is compact. For the definitions of locally compact quantum groups such as the commutant $\mathbb{G}^{\prime}$, the opposite $\mathbb{G}^{o p}$ etc., we refer the readers to [10, Section 4].

It is known that every locally compact group $\Gamma$ canonically gives rise to a commutative locally compact quantum group whose underlying von Neumann algebra is $L^{\infty}(\Gamma)$. We denote it by $\mathbb{G}(\Gamma)$. The underlying von Neumann algebra of the dual $\widehat{\mathbb{G}}(\Gamma)$ is the group von Neumann algebra of $\Gamma$ generated by the left regular representation of $\Gamma$.

We denote by $I G(\mathbb{G})$ the set of all unitaries $u \in M$ satisfying $\Delta(u)=u \otimes u$. The group $I G(\mathbb{G})$ is called the intrinsic group of $\mathbb{G}$. Next define $\mathcal{G}(\mathbb{G})$ to be the group of all automorphisms $\beta$ of $M$ such that $(\beta \otimes i d) \circ \Delta=\Delta \circ \beta$. By [2] (see [19] also), $I G(\widehat{\mathbb{G}})$ is topologically isomorphic to $\mathcal{G}(\mathbb{G})$ through the mapping: $v \in$ $I G(\widehat{\mathbb{G}}) \longmapsto \beta_{v}:=\left.\operatorname{Ad} v\right|_{M} \in \mathcal{G}(\mathbb{G})$. Here $I G(\widehat{\mathbb{G}})$ is endowed with the strong-operator topology, and, for a general (separable) von Neumann algebra $P$, we consider on the automorphism group Aut $(P)$ of $P$ the topology of simple convergence on the predual. It is known that $v$ is the canonical implementation of $\beta_{v}$.

We say that $N \subseteq M$ is a right co-ideal (von Neumann subalgebra) of $\mathbb{G}$ if $N$ is a von Neumann subalgebra of $M$ satisfying $\Delta(N) \subseteq N \otimes M$. Thanks to 4 . Théorème 3.3], we know that $N \subseteq M$ is a right co-ideal of $\mathbb{G}$ if and only if one has

$$
N=M \cap\left(\widehat{M} \cap N^{\prime}\right)^{\prime} .
$$

A (left) action of $\mathbb{G}$ on a von Neumann algebra $A$ is a normal injective unital *-homomorphism $\alpha$ from $A$ into $M \otimes A$ satisfying $\left(i d_{M} \otimes \alpha\right) \circ \alpha=\left(\Delta \otimes i d_{M}\right) \circ \alpha$ ([16]).

Fix an action $\alpha$ of $\mathbb{G}$ on a von Neumann algebra $A$. By [16, Proposition 1.3], the equation

$$
T_{\alpha}(a):=(\psi \otimes i d)(\alpha(a)) \quad\left(a \in A_{+}\right)
$$


defines a faithful normal operator-valued weight $T_{\alpha}$ from $A$ onto $A^{\alpha}:=\{a \in A$ : $\alpha(a)=1 \otimes a\}$, the fixed-point algebra $A^{\alpha}$ of $\alpha$. We call $T_{\alpha}$ the operator-valued weight associated to the action $\alpha$.

The crossed product of $A$ by the action $\alpha$ is by definition the von Neumann algebra generated by $\alpha(A)$ and $\widehat{M} \otimes \mathbf{C}$. We denote it by $\mathbb{G}_{\alpha} \ltimes A$. By 16 , Proposition 2.2], there exists a unique action $\hat{\alpha}$ of $\widehat{\mathbb{G}}^{o p}$ on $\mathbb{G}_{\alpha} \ltimes A$, called the dual action of $\alpha$, such that

$$
\left(\mathbb{G}_{\alpha} \ltimes A\right)^{\hat{\alpha}}=\alpha(A), \quad \hat{\alpha}(z \otimes 1)=\hat{\Delta}^{o p}(z) \otimes 1 \quad(z \in \widehat{M}) .
$$

For every faithful normal semifinite weight $\phi$ on $A$, by using the operator-valued weight $T_{\hat{\alpha}}$ associated to the dual action $\hat{\alpha}$, the equation

$$
\tilde{\phi}:=\phi \circ \alpha^{-1} \circ T_{\hat{\alpha}}
$$

defines a faithful normal semifinite weight $\tilde{\phi}$ on $\mathbb{G}_{\alpha} \ltimes A$. The weight $\tilde{\phi}$ is called the dual weight of $\phi$. The Hilbert space $H_{\tilde{\phi}}$ is identified with $H_{\varphi} \otimes H_{\phi}$. The unitary $U_{\phi}$ on $H_{\varphi} \otimes H_{\phi}$ defined by

$$
U_{\phi}:=J_{\tilde{\phi}}\left(\hat{J} \otimes J_{\phi}\right)
$$

is called the canonical implementation of $\alpha$ (see [16]). It satisfies

$$
\alpha(a)=U_{\phi}(1 \otimes a) U_{\phi}^{*} \quad(a \in A) .
$$

By [16. Theorem 2.6], there is a unital $*$-isomorphism $\Theta$ from the double crossed product $\widehat{\mathbb{G}}^{o p}{ }_{\hat{\alpha}} \ltimes\left(\mathbb{G}_{\alpha} \ltimes A\right)$ onto $B\left(H_{\varphi}\right) \otimes A$, and an action $\tilde{\alpha}$ of $\mathbb{G}$ on $B\left(H_{\varphi}\right) \otimes A$ such that

$$
\begin{aligned}
& \tilde{\alpha}:=\operatorname{Ad}\left(\Sigma V^{*} \Sigma \otimes 1\right) \circ\left(\sigma \otimes i d_{A}\right) \circ\left(i d_{B\left(H_{\varphi}\right)} \otimes \alpha\right), \\
& \quad(\operatorname{Ad}(J \hat{J}) \otimes \Theta) \circ \hat{\hat{\alpha}}=\tilde{\alpha} \circ \Theta,
\end{aligned}
$$

where $V:=(\hat{J} \otimes \hat{J}) \Sigma W^{*} \Sigma(\hat{J} \otimes \hat{J})$ and $\Sigma: H_{\varphi} \otimes H_{\varphi} \rightarrow H_{\varphi} \otimes H_{\varphi}$ is the flip. We call $\tilde{\alpha}$ the stabilization of $\alpha$.

We say that the action $\alpha$ is integrable if $T_{\alpha}$ is semifinite. The action $\alpha$ is said to be minimal if $A \cap\left(A^{\alpha}\right)^{\prime}=\mathbf{C}$ and the linear span of $\left\{(i d \otimes \omega)(\alpha(a)): a \in A, \omega \in A_{*}\right\}$ is $\sigma$-weakly dense in $M$.

Finally, $\mathbb{G}$ is called a Kac algebra if $\tau_{t}=i d$ and $\sigma^{\phi}=\sigma^{\psi}$. For the general theory of Kac algebras, refer to [6].

\section{Realization of intrinsic groups in $\operatorname{Aut}\left(A / A^{\alpha}\right)$}

Given a von Neumann algebra $P$ and a von Neumann subalgebra $Q$ of $P$, we define Aut $(P / Q)$ to be the group of all automorphisms of $P$ leaving $Q$ invariant pointwise.

Let $\mathbb{G}=(M, \Delta, \varphi, \psi)$ be a locally compact quantum group. Suppose now that we have an action $\alpha$ of $\mathbb{G}$ on a von Neumann algebra $A$. Throughout this paper, $A$ is always assumed to be a non-type $I_{n}$ factor $(n \in \mathbf{N})$.

The mapping considered in the following proposition is essentially observed by Enock and Schwartz in [5] as a special case of their constructions of certain morphisms associated to an action of a Kac algebra. The mapping is still defined even for a general locally compact quantum group. 
Proposition 3.1. There exists a unique continuous homomorphism from $\mathcal{G}(\mathbb{G})$ into Aut $\left(A / A^{\alpha}\right)$ such that, with $\theta_{\beta}$ the image of $\beta \in \mathcal{G}(\mathbb{G})$ under this homomorphism, we have

$$
(\beta \otimes i d) \circ \alpha=\alpha \circ \theta_{\beta} .
$$

If the action $\alpha$ enjoys the property that the linear span of $\left\{\left(i d_{M} \otimes \omega\right)(\alpha(a)): a \in\right.$ $\left.A, \omega \in A_{*}\right\}$ is $\sigma$-weakly dense in $M$, then the above homomorphism is injective.

Proof. Let $\beta \in \mathcal{G}(\mathbb{G})$ and $a \in A$. Set $X:=\left(\beta \otimes i d_{A}\right) \circ \alpha(a) \in M \otimes A$. Then

$$
\begin{aligned}
\left(i d_{M} \otimes \alpha\right)(X) & =\left(i d_{M} \otimes \alpha\right) \circ\left(\beta \otimes i d_{A}\right) \circ \alpha(a) \\
& =\left(\beta \otimes i d_{M} \otimes i d_{A}\right) \circ\left(i d_{M} \otimes \alpha\right) \circ \alpha(a) \\
& =\left(\beta \otimes i d_{M} \otimes i d_{A}\right) \circ\left(\Delta \otimes i d_{A}\right) \circ \alpha(a) \\
& =\left(\Delta \otimes i d_{A}\right) \circ\left(\beta \otimes i d_{A}\right) \circ \alpha(a)=\left(\Delta \otimes i d_{A}\right)(X) .
\end{aligned}
$$

From [16 Theorem 2.7], there exists a unique element $\theta_{\beta}(a) \in A$ such that

$$
\left(\beta \otimes i d_{A}\right) \circ \alpha(a)=X=\alpha\left(\theta_{\beta}(a)\right) .
$$

It is easy to check by using (3.1) that $\theta_{\beta}$ is a homomorphism from $A$ into itself. Moreover, one can easily verify that $\theta_{\beta_{1} \beta_{2}}=\theta_{\beta_{1}} \circ \theta_{\beta_{2}}$ and $\theta_{i d}=i d$. Hence $\theta_{\beta}$ is an automorphism of $A$. That the restriction of $\theta_{\beta}$ to $A^{\alpha}$ is the identity follows also from (3.1). Thus the mapping $\beta \in \mathcal{G}(\mathbb{G}) \mapsto \theta_{\beta} \in \operatorname{Aut}\left(A / A^{\alpha}\right)$ is indeed a homomorphism. Because of (3.1), we find that $\left.\left(\beta \otimes i d_{A}\right)\right|_{\alpha(A)}$ is an automorphism. With this in mind, $\theta_{\beta}$ has the form

$$
\theta_{\beta}=\left.\alpha^{-1} \circ\left(\beta \otimes i d_{A}\right)\right|_{\alpha(A)} \circ \alpha .
$$

Hence $\beta \longmapsto \theta_{\beta}$ is continuous.

Now suppose that the linear span of $\left\{\left(i d_{M} \otimes \omega\right)(\alpha(a)): a \in A, \omega \in A_{*}\right\}$ is $\sigma$-weakly dense in $M$. If $\theta_{\beta}=i d$, then (3.1) implies that $\beta$ is the identity on $\left\{\left(i d_{M} \otimes \omega\right)(\alpha(a)): a \in A, \omega \in A_{*}\right\}$. So $\beta=i d$. Consequently, the homomorphism in question is injective.

Lemma 3.2. Let $\beta$ be an automorphism of $M$ such that there is a $\theta \in \operatorname{Aut}(A)$ such that $(\beta \otimes i d) \circ \alpha=\alpha \circ \theta$. Suppose that the linear span of $\left\{\left(i d_{M} \otimes \omega\right)(\alpha(a))\right.$ : $\left.a \in A, \omega \in A_{*}\right\}$ is $\sigma$-weakly dense in $M$. Then $\beta$ belongs to $\mathcal{G}(\mathbb{G})$, and one has $\theta=\theta_{\beta}$.

Proof. Note first that, if $\theta$ is an automorphism as above, then it automatically belongs to Aut $\left(A / A^{\alpha}\right)$. Therefore, it suffices by Proposition 3.1 to show that $\beta$ belongs to $\mathcal{G}(\mathbb{G})$.

Let $a \in A$ and $\omega \in A_{*}$. Then we have

$$
\begin{aligned}
(\beta \otimes & \left.i d_{M}\right) \circ \Delta\left(\left(i d_{M} \otimes \omega\right)(\alpha(a))\right) \\
& \left.=\left(\beta \otimes i d_{M}\right) \circ\left(i d_{M} \otimes i d_{M} \otimes \omega\right) \circ\left(\Delta \otimes i d_{A}\right)(\alpha(a))\right) \\
& =\left(i d_{M} \otimes i d_{M} \otimes \omega\right) \circ\left(\beta \otimes i d_{M} \otimes i d_{A}\right) \circ\left(i d_{M} \otimes \alpha\right)(\alpha(a)) \\
& =\left(i d_{M} \otimes i d_{M} \otimes \omega\right) \circ\left(i d_{M} \otimes \alpha\right) \circ\left(\beta \otimes i d_{A}\right)(\alpha(a)) \\
& =\left(i d_{M} \otimes i d_{M} \otimes \omega\right) \circ\left(i d_{M} \otimes \alpha\right) \circ \alpha(\theta(a)) \\
& =\left(i d_{M} \otimes i d_{M} \otimes \omega\right) \circ\left(\Delta \otimes i d_{A}\right) \circ \alpha(\theta(a)) \\
& =\Delta \circ \beta\left(\left(i d_{M} \otimes \omega\right)(\alpha(a))\right) .
\end{aligned}
$$

From our assumption on $\alpha$, it follows that $\beta$ satisfies $(\beta \otimes i d) \circ \Delta=\Delta \circ \beta$. 
Let $\iota$ be the trivial action of $\mathbb{G}$ on $\mathbf{C}$. Namely, $\iota$ is the mapping from $\mathbf{C}$ into $M \otimes \mathbf{C}$ defined by $\iota(c):=1 \otimes c \quad(c \in \mathbf{C})$. Then the crossed product $\mathbb{G}_{\iota} \ltimes \mathbf{C}$ is (canonically isomorphic to) $\widehat{M}$, and the dual action $\hat{\iota}$ is the coproduct $\hat{\Delta}^{o p}$. It is also clear that the dual weight of $t_{\mathbf{C}}$ corresponds to $\hat{\varphi}$. In this case, the stabilization $\tilde{\iota}$ of $\iota$ has the form $\tilde{\iota}(x)=\Sigma V^{*} \Sigma(1 \otimes x) \Sigma V \Sigma$ for any $x \in B\left(H_{\varphi}\right)$. Therefore, we obtain

$$
\tilde{\alpha}(x \otimes 1)=\tilde{\iota}(x) \otimes 1
$$

for any $x \in B\left(H_{\varphi}\right)$.

Lemma 3.3. Let $z$ be in $B\left(H_{\varphi}\right)$ such that $z \otimes 1 \in \mathbb{G}_{\alpha} \ltimes A$. Then $z$ belongs to $\widehat{M}$.

Proof. Let $z$ be an element as above. Since $\left(B\left(H_{\varphi}\right) \otimes A\right)^{\tilde{\alpha}}=\mathbb{G}_{\alpha} \ltimes A$, we have $\tilde{\alpha}(z \otimes 1)=1 \otimes z \otimes 1$. On the other hand, by (3.2), we have $\tilde{\alpha}(z \otimes 1)=\tilde{\imath}(z) \otimes 1$. Hence we obtain $\tilde{\iota}(z)=1 \otimes z$. Since $B\left(H_{\varphi}\right)^{\tilde{\tau}}=\widehat{M}, z$ must belong to $\widehat{M}$.

Lemma 3.4. Suppose that $\alpha$ is minimal, Then we have $\alpha(A)^{\prime} \cap B\left(H_{\varphi}\right) \otimes A=$ $M^{\prime} \otimes \mathbf{C}$.

Proof. It is clear that $M^{\prime} \otimes \mathbf{C}$ is contained in $\alpha(A)^{\prime} \cap B\left(H_{\varphi}\right) \otimes A$. Take any $T \in \alpha(A)^{\prime} \cap B\left(H_{\varphi}\right) \otimes A$. Since $T$ particularly commutes with any element of $\alpha\left(A^{\alpha}\right)=\mathbf{C} \otimes A^{\alpha}$, it follows from the minimality of $\alpha$ that $T$ belongs to $B\left(H_{\varphi}\right) \otimes \mathbf{C}$. So it has the form $T=y \otimes 1$ for some $y \in B\left(H_{\varphi}\right)$. If $a \in A$ and $\omega \in A_{*}$, then we have

$$
y(i d \otimes \omega)(\alpha(a))=(i d \otimes \omega)(T \alpha(a))=(i d \otimes \omega)(\alpha(a) T)=(i d \otimes \omega)(\alpha(a)) y .
$$

By minimality of $\alpha, y$ must be in $M^{\prime}$.

Since $\left(\mathbb{G}_{\alpha} \ltimes A\right)^{\hat{\alpha}}=\alpha(A)$, it follows from Proposition 3.1 that there exists a continuous homomorphism $\beta \in \mathcal{G}\left(\widehat{\mathbb{G}}^{o p}\right) \longmapsto \theta_{\beta} \in \operatorname{Aut}\left(\mathbb{G}_{\alpha} \ltimes A / \alpha(A)\right)$ satisfying $(\beta \otimes i d) \circ \hat{\alpha}=\hat{\alpha} \circ \theta_{\beta}$. Since $\hat{\alpha}$ enjoys the property mentioned in Proposition 3.1] the homomorphism $\beta \longmapsto \theta_{\beta}$ is injective in this case.

Lemma 3.5. If the action $\alpha$ is minimal, then the homomorphism $\beta \in \mathcal{G}\left(\widehat{\mathbb{G}}^{o p}\right) \longmapsto$ $\theta_{\beta} \in \operatorname{Aut}\left(\mathbb{G}_{\alpha} \ltimes A / \alpha(A)\right)$ is a topological isomorphism.

Proof. Let $\theta$ be in Aut $\left(\mathbb{G}_{\alpha} \ltimes A / \alpha(A)\right)$. If $z \in \widehat{M}$ and $b \in A^{\alpha}$, then

$$
\begin{aligned}
\theta(z \otimes 1)(1 \otimes b) & =\theta(z \otimes 1) \alpha(b)=\theta((z \otimes 1) \alpha(b))=\theta(z \otimes b) \\
& =\theta(\alpha(b)(z \otimes 1))=(1 \otimes b) \theta(z \otimes 1) .
\end{aligned}
$$

This shows that $\theta(\widehat{M} \otimes \mathbf{C}) \subseteq\left(\mathbf{C} \otimes A^{\alpha}\right)^{\prime}=B\left(H_{\varphi}\right) \otimes\left(A^{\alpha}\right)^{\prime}$. From this, we obtain

$$
\theta(\widehat{M} \otimes \mathbf{C}) \subseteq B\left(H_{\varphi}\right) \otimes\left\{A \cap\left(A^{\alpha}\right)^{\prime}\right\}=B\left(H_{\varphi}\right) \otimes \mathbf{C} .
$$

Hence, for any $z \in \widehat{M}$, there is a unique $\beta_{\theta}(z) \in B\left(H_{\varphi}\right)$ such that

$$
\theta(z \otimes 1)=\beta_{\theta}(z) \otimes 1 .
$$

Thanks to Lemma 3.3, $\beta_{\theta}(z)$ belongs to $\widehat{M}$. Due to (3.3), it is easy to see that $\beta_{\theta}$ is an automorphism of $\widehat{M}$, and that $\beta_{\theta_{1} \theta_{2}}=\beta_{\theta_{1}} \circ \beta_{\theta_{2}}, \beta_{i d}=i d$.

If $a \in A$, then

$$
\left(\beta_{\theta} \otimes i d\right)(\hat{\alpha}(\alpha(a)))=\left(\beta_{\theta} \otimes i d\right)(1 \otimes \alpha(a))=1 \otimes \alpha(a)=\hat{\alpha}(\theta(\alpha(a))) .
$$


Fix a faithful normal semifinite weight $\omega$ on $A$, and regard $A$ as represented on $H_{\omega}$. Let $\tilde{J}_{\omega}$ be the modular conjugation of the dual weight $\tilde{\omega}$ and $U$ the canonical implementation of $\alpha$ on $H_{\varphi} \otimes H_{\omega}$. So $U=\tilde{J}_{\omega}\left(J \otimes J_{\omega}\right)$. Choose the canonical implementation unitary $V$ of $\theta$ on $H_{\varphi} \otimes H_{\omega}$. Then we have $V \in \alpha(A)^{\prime}$. Since $V$ commutes with $\tilde{J}_{\omega}$, we also have $V \in \tilde{J}_{\omega} \alpha(A)^{\prime} \tilde{J}_{\omega}=\left(\mathbf{C} \otimes J_{\omega} A J_{\omega}\right)^{\prime}=B\left(H_{\varphi}\right) \otimes A$. It follows from Lemma 3.4 that there exists a unitary $v \in M^{\prime}$ such that $V=v \otimes 1$. Therefore we have $\beta_{\theta}(z)=v z v^{*}$ for any $z \in \widehat{M}$. By [12, Proposition I.9], which is valid also for any locally compact quantum group, we see that $r v$ belongs to $I G\left(\mathbb{G}^{\prime}\right)$ for some $r \in \mathbf{C}$ with $|r|=1$. Since both $V$ and $r V$ are the canonical implementation of $\theta$, we must have $r=1$. So $v$ is in $I G\left(\mathbb{G}^{\prime}\right)$. It follows that $\beta_{\theta}$ belongs to $\mathcal{G}\left(\widehat{\mathbb{G}}^{o p}\right)$. Let $z \in \widehat{M}$. Then we have

$$
\begin{aligned}
\hat{\alpha}(\theta(z \otimes 1)) & =\hat{\alpha}\left(\beta_{\theta}(z) \otimes 1\right)=\hat{\Delta}^{o p}\left(\beta_{\theta}(z)\right) \otimes 1 \\
& =\left(\beta_{\theta} \otimes i d\right) \circ \hat{\Delta}^{o p}(z) \otimes 1=\left(\beta_{\theta} \otimes i d\right) \circ \hat{\alpha}(z \otimes 1) .
\end{aligned}
$$

From this, together with (3.4), we get $\left(\beta_{\theta} \otimes i d\right) \circ \hat{\alpha}=\hat{\alpha} \circ \theta$. By Lemma 3.2, we find that $\theta=\theta_{\beta_{\theta}}$. Thus we have shown the surjectivity. The inverse map is also continuous due to (3.3).

Theorem 3.6. If $\alpha$ is a minimal, integrable action of a locally compact quantum group $\mathbb{G}=(M, \Delta, \varphi, \psi)$ on a factor $A$, then there exists a topological isomorphism $\beta \in \mathcal{G}(\mathbb{G}) \longmapsto \theta_{\beta} \in \operatorname{Aut}\left(A / A^{\alpha}\right)$ with the property $(\beta \otimes i d) \circ \alpha=\alpha \circ \theta_{\beta}$.

Proof. If $A^{\alpha}$ is infinite, then, by [16, Proposition 6.4], $\alpha$ is a dual action. Hence the assertion follows from Lemma 3.5 .

To deal with a general case, take a (separable) infinite factor $L$, and put $\bar{A}:=$ $L \otimes A$. Also define $\bar{\alpha}:=(\sigma \otimes i d) \circ\left(i d_{L} \otimes \alpha\right)$, which is an action of $\mathbb{G}$ on $\bar{A}$ with $\bar{A}^{\bar{\alpha}}=L \otimes A^{\alpha}$ infinite. Remark that $\operatorname{Aut}\left(\bar{A} / \bar{A}^{\bar{\alpha}}\right)=\left\{i d_{L} \otimes \theta: \theta \in \operatorname{Aut}\left(A / A^{\alpha}\right)\right\}$. Let $\theta \in \operatorname{Aut}\left(A / A^{\alpha}\right)$. By the previous paragraph and the above remark, there exists $\beta \in \mathcal{G}(\mathbb{G})$ such that $(\beta \otimes i d) \circ \bar{\alpha}=\bar{\alpha} \circ\left(i d_{L} \otimes \theta\right)$. But this yields $(\beta \otimes i d) \circ \alpha=\alpha \circ \theta$.

\section{Regularity of $A^{\alpha}$ IN $A$}

As in the previous section, let $\alpha$ be a minimal action of a locally compact quantum group $\mathbb{G}=(M, \Delta, \varphi, \psi)$ on a factor $A$.

We represent $A$ on a (separable) Hilbert space $K$ so that $\left\{A, K, J_{A}\right\}$ is a standard representation. Let $u \in \mathcal{N}\left(A^{\alpha}\right)$. Then the restriction of $\operatorname{Ad} u$ to $\left(A^{\alpha}\right)^{\prime}$ clearly defines an automorphism $\theta_{u}$ in Aut $\left(\left(A^{\alpha}\right)^{\prime} / A^{\prime}\right)$. The homomorphism $u \in \mathcal{N}\left(A^{\alpha}\right) \longmapsto \theta_{u} \in$ Aut $\left(\left(A^{\alpha}\right)^{\prime} / A^{\prime}\right)$ obviously has $\mathcal{U}\left(A^{\alpha}\right)$ as its kernel. The basic extension for $A^{\alpha} \subseteq A$ is denoted by $A_{1}$. So we have $A_{1}=J_{A}\left(A^{\alpha}\right)^{\prime} J_{A}$. If $A^{\alpha}$ is infinite, then so is $A$. Thanks to [3. Corollaire 1], we may then choose the above Hilbert space $K$ in such a way that there is a unit vector $\xi_{0} \in K$ that is cyclic and separating for both $A$ and $A^{\alpha}$. Let $J_{A}$ then denote the modular conjugation of $A$ associated to $\xi_{0}$.

Lemma 4.1. Suppose as above that $A^{\alpha}$ is infinite. Then the homomorphism $u \in \mathcal{N}\left(A^{\alpha}\right) \longmapsto \theta_{u} \in$ Aut $\left(\left(A^{\alpha}\right)^{\prime} / A^{\prime}\right)$ defined above is surjective, that is, it has Aut $\left(\left(A^{\alpha}\right)^{\prime} / A^{\prime}\right)$ as its image.

Proof. Let $\theta$ be in Aut $\left(\left(A^{\alpha}\right)^{\prime} / A^{\prime}\right)$. Since $\left\{\left(A^{\alpha}\right)^{\prime}, K\right\}$ is a standard representation, there exists a unitary $v$ on $K$ such that $\theta=\left.\operatorname{Ad} v\right|_{\left(A^{\alpha}\right)^{\prime}}$. Since $\left.\theta\right|_{A^{\prime}}=i d$, $v$ belongs to $\left(A^{\prime}\right)^{\prime}=A$. It is easy to see that $v$ is in $\mathcal{N}\left(A^{\alpha}\right)$. We clearly have $\theta=\theta_{v}$. 
Remark. If $A$ is finite and $\mathbb{G}$ is finite-dimensional (so that the Jones index $\left[A: A^{\alpha}\right]$ is finite), then it follows from [11, Proposition 1.7] that the map $u \in \mathcal{N}\left(A^{\alpha}\right) \longmapsto$ $\theta_{u} \in \operatorname{Aut}\left(\left(A^{\alpha}\right)^{\prime} / A^{\prime}\right)$ is surjective.

From now on, we assume that $\alpha$ is minimal and integrable ( $A$ is not necessarily infinite). Fix a faithful normal semifinite weight $\omega$ on $A$, and represent $A$ on $H_{\omega}$ now. Let $U$ be the canonical implementation of $\alpha$ associated to $\omega$. Due to [16. Proposition 6.2], this assumption is equivalent to the one that $\alpha$ is outer and integrable. From [16, Corollary 5.6] and [16, Proposition 6.2], it follows that the inclusions

$$
\mathbf{C} \otimes A^{\alpha} \subseteq \alpha(A) \subseteq \mathbb{G}_{\alpha} \ltimes A \quad \text { and } \quad A^{\alpha} \subseteq A \subseteq A_{1}
$$

are isomorphic. According to [16, Corollary 5.6], the isomorphism $\rho: \mathbb{G}_{\alpha} \ltimes A \longrightarrow A_{1}$ is characterized by

$$
\begin{aligned}
\rho(\alpha(a)) & =a & & (a \in A), \\
\rho((\phi \otimes i d)(W) \otimes 1) & =(\phi \otimes i d)\left(U^{*}\right) & & \left(\phi \in M_{*}\right) .
\end{aligned}
$$

Incidentally, Equation (4.2) can be rewritten as

$$
\rho\left(\lambda^{\prime}(\phi) \otimes 1\right)=(\hat{J} J \phi J \hat{J} \otimes i d)(U) \quad\left(\phi \in\left(M^{\prime}\right)_{*}\right),
$$

where $\lambda^{\prime}$ stands for the left regular representation of $\mathbb{G}^{\prime}$ that is given by $\lambda^{\prime}(\phi)=$ $\lambda\left(\hat{J} J \phi^{*} J \hat{J}\right)^{*}$. We will make use of this isomorphism in the discussion that follows. Since $A_{1}=J_{\omega}\left(A^{\alpha}\right)^{\prime} J_{\omega}$ and $A=J_{\omega} A^{\prime} J_{\omega}$, Aut $\left(\left(A^{\alpha}\right)^{\prime} / A^{\prime}\right)$ is isomorphic to Aut $\left(\mathbb{G}_{\alpha} \ltimes\right.$ $A / \alpha(A))$. Thus we obtain a homomorphism from $\mathcal{N}\left(A^{\alpha}\right)$ into Aut $\left(\mathbb{G}_{\alpha} \ltimes A / \alpha(A)\right)$. By using the isomorphism $\rho$ introduced above, it is explicitly given as follows: $u \in \mathcal{N}\left(A^{\alpha}\right) \longmapsto \theta_{u}:=\rho^{-1} \circ \operatorname{Ad}\left(J_{\omega} u J_{\omega}\right) \mid A_{1} \circ \rho$. As we saw in Lemma 4.1 and the remark after it, this homomorphism is surjective if $A^{\alpha}$ is infinite or if $A$ is finite and $\mathbb{G}$ is finite-dimensional. But it may not be in general. So our next goal is to identify its image in detail. For this, first note that, thanks to Proposition 3.5, it is enough to identify automorphisms $\beta \in \mathcal{G}\left(\widehat{\mathbb{G}}^{o p}\right)$ such that $\theta_{\beta}=\theta_{u}$ for $u \in \mathcal{N}\left(A^{\alpha}\right)$. Moreover, since each $\theta_{\beta}$ has the form $\theta_{\beta}=\operatorname{Ad}(v \otimes 1)$ for a unique $v \in I G\left(\mathbb{G}^{\prime}\right)$ with $\beta=\operatorname{Ad} v$, it suffices to identify unitaries $v \in I G\left(\mathbb{G}^{\prime}\right)$ such that $\operatorname{Ad}(v \otimes 1)=\theta_{u}$ for some $u \in \mathcal{N}\left(A^{\alpha}\right)$.

Proposition 4.2. Let $\alpha$ be a minimal and integrable action of a locally compact quantum group $\mathbb{G}=(M, \Delta, \varphi, \psi)$ on a factor $A$.

(1) For any $u \in \mathcal{N}\left(A^{\alpha}\right)$, there exists a unique unitary $w(u) \in I G(\mathbb{G})$ such that $\alpha(u)\left(1 \otimes u^{*}\right)=w(u) \otimes 1$

(2) For any $u \in \mathcal{N}\left(A^{\alpha}\right)$, with $w(u)$ in Part (1), we have

$$
\theta_{u}=\operatorname{Ad}(J w(u) J \otimes 1)=\operatorname{Ad}\left(J \otimes J_{\omega}\right) \alpha(u)\left(1 \otimes u^{*}\right)\left(J \otimes J_{\omega}\right) .
$$

We denote the unitary $J w(u) J$ in $I G\left(\mathbb{G}^{\prime}\right)$ by $v(u)$.

Proof. Let $u \in \mathcal{N}\left(A^{\alpha}\right)$.

(1) It is straightforward to check that $\alpha(u)\left(1 \otimes u^{*}\right)$ commutes with any element of the form $1 \otimes a$, where $a \in A^{\alpha}$. So it is contained in $M \otimes A \cap\left(\mathbf{C} \otimes A^{\alpha}\right)^{\prime}=M \otimes \mathbf{C}$. Hence there exists a unitary $w \in M$ such that $\alpha(u)\left(1 \otimes u^{*}\right)=w \otimes 1$. Thus $\alpha(u)=w \otimes u$. By applying $\Delta \otimes i d$ to both sides of this identity, we obtain $\Delta(w)=w \otimes w$. Therefore $w$ belongs to $I G(\mathbb{G})$. So put $w(u):=w$. 
(2) Choose $\beta \in \mathcal{G}\left(\widehat{\mathbb{G}}^{o p}\right)$ and $v \in I G\left(\mathbb{G}^{\prime}\right)$ such that $\theta_{u}=\theta_{\beta}$ and $\beta=\operatorname{Ad} v$. Since $\widehat{\mathbb{G}^{\prime}}=\widehat{\mathbb{G}}^{o p}$, we have $\beta\left(\lambda^{\prime}(\phi)\right)=\lambda^{\prime}(v \phi)$. Therefore, by (4.3), the identity $\theta_{u}=\theta_{\beta}$ is equivalent to the next:

$$
\operatorname{Ad}\left(J_{\omega} u J_{\omega}\right)(\hat{J} J \phi J \hat{J} \otimes i d)(U)=(\hat{J} J v \phi J \hat{J} \otimes i d)(U) \quad\left(\phi \in\left(M^{\prime}\right)_{*}\right) .
$$

This is further equivalent to

$$
\left(J \hat{J} \otimes J_{\omega} u J_{\omega}\right) U\left(J \hat{J} \otimes J_{\omega} u^{*} J_{\omega}\right)=(J \hat{J} \otimes 1) U(J \hat{J} v \otimes 1) .
$$

By using $U^{*}=\left(\hat{J} \otimes J_{\omega}\right) U\left(\hat{J} \otimes J_{\omega}\right)$, we can reduce the above identity to

$$
\alpha(u)\left(1 \otimes u^{*}\right)=J v J \otimes 1 .
$$

Consequently, we obtain $w(u)=J v J$.

Definition 4.3. Let $\beta$ be an action of $\mathbb{G}$ on a von Neumann algebra $P$. For a right co-ideal $N$ of $\mathbb{G}$, the intermediate von Neumann subalgebra $P(N)$ of $P^{\beta} \subseteq P$ associated to $N$ (see [7]) is defined by

$$
P(N):=\{x \in P: \beta(x) \in N \otimes P\} .
$$

The following lemma is proven in [4] for the case where $\mathbb{G}$ is a Woronowicz algebra. The claim and its proof are still valid even for a general locally compact quantum group.

Lemma 4.4 (Proposition 3.5, 4]). Let $N$ be a right co-ideal of $\mathbb{G}$. Then we have

$$
N=\left\{(i d \otimes \omega)(\Delta(x)): \omega \in M_{*}, a \in N\right\}^{\prime \prime} .
$$

Proof. Denote by $N_{1}$ the right-hand side of the above claim. Clearly we have $N_{1} \subseteq N$. Let $x \in N$. For any $y \in N_{1}^{\prime}, \rho \in B\left(H_{\varphi}\right)_{*}$ and $\omega \in M_{*}$, we have

$$
\begin{aligned}
(\rho \otimes \omega)((y \otimes 1) \Delta(x)) & =\rho(y(i d \otimes \omega)(\Delta(x))) \\
& =\rho((i d \otimes \omega)(\Delta(x)) y)=(\rho \otimes \omega)(\Delta(x)(y \otimes 1)) .
\end{aligned}
$$

This shows that $\Delta(x)$ is in $N_{1} \otimes M$. Hence $\Delta(N) \subseteq N_{1} \otimes M$. In particular, $N_{1}$ is also a right co-ideal of $\mathbb{G}$. Therefore, $\gamma:=\left.\Delta^{o p}\right|_{N_{1}}$ defines an action $\gamma$ of $\mathbb{G}^{o p}$ on $N_{1}$. If $x \in N$, then, by the above result, $\Delta^{o p}(x) \in M \otimes N_{1}$. Moreover, we have

$$
(i d \otimes \gamma)\left(\Delta^{o p}(x)\right)=\left(i d \otimes \Delta^{o p}\right)\left(\Delta^{o p}(x)\right)=\left(\Delta^{o p} \otimes i d\right)\left(\Delta^{o p}(x)\right) .
$$

From [16, Theorem 2.7], it follows that $\Delta^{o p}(x)$ belongs to $\gamma\left(N_{1}\right)=\Delta^{o p}\left(N_{1}\right)$. Thus $x \in N_{1}$.

Lemma 4.5. Let $\beta$ be an action of $\mathbb{G}$ on a von Neumann algebra $P$. For any intermediate von Neumann subalgebra $Q$ of $P^{\alpha} \subseteq P,\{(i d \otimes \omega)(\beta(x)): x \in Q, \omega \in$ $\left.P_{*}\right\}^{\prime \prime}$ is a right co-ideal of $\mathbb{G}$. We denote this right co-ideal by $N_{\beta}(Q)$, and call it the right co-ideal associated to $Q$.

Proof. Let $y \in N_{\beta}(Q)^{\prime}$. For any $\rho, \phi \in B\left(H_{\varphi}\right), \omega \in P_{*}$ and $x \in Q$, we have

$$
\begin{aligned}
\langle(y \otimes 1) \Delta((i d \otimes \omega)(\beta(x))), \rho \otimes \phi\rangle & \\
& =\langle(y \otimes 1)(i d \otimes i d \otimes \omega)((i d \otimes \beta)(\beta(x))), \rho \otimes \phi\rangle \\
& =\langle y(i d \otimes(\phi \otimes \omega) \circ \beta)(\beta(x)), \rho\rangle \\
& =\langle(i d \otimes(\phi \otimes \omega) \circ \beta)(\beta(x)) y, \rho\rangle \\
& =\langle\Delta((i d \otimes \omega)(\beta(x)))(y \otimes 1), \rho \otimes \phi\rangle .
\end{aligned}
$$


From this, we see that $\Delta((i d \otimes \omega)(\beta(x)))$ is included in $N_{\beta}(Q) \otimes M$. Hence $N_{\beta}(Q)$ is a right co-ideal of $\mathbb{G}$.

Lemma 4.6. Let $\beta$ be an action of $\mathbb{G}$ on a von Neumann algebra $P$. With the notation introduced above, we have $N=N_{\hat{\beta}}\left(\left(\mathbb{G}_{\beta} \ltimes P\right)(N)\right)$ for any right co-ideal $N$ of $\widehat{\mathbb{G}}^{o p}$. If $L$ is the von Neumann subalgebra of $\mathbb{G}_{\beta} \ltimes P$ generated by $\beta(P)$ and $N \otimes \mathbf{C}$ for some right co-ideal $N$ of $\widehat{\mathbb{G}}^{o p}$, then $N_{\hat{\beta}}(L)=N$.

Proof. Let $N$ be a right co-ideal of $\widehat{\mathbb{G}}^{o p}$. It is plain to see that $N_{\hat{\beta}}\left(\left(\mathbb{G}_{\beta} \ltimes P\right)(N)\right)$ is contained in $N$. Let $x \in N$ and $\rho \in B\left(H_{\varphi}\right)_{*}$. Since $\hat{\Delta}^{o p}(x) \in N \otimes \widehat{M}$, we have

$$
\hat{\beta}(x \otimes 1)=\hat{\Delta}^{o p}(x) \otimes 1 \in N \otimes\left(\mathbb{G}_{\beta} \ltimes P\right) .
$$

Thus $x \otimes 1$ belongs to $\left(\mathbb{G}_{\beta} \ltimes P\right)(N)$. If $\omega \in P_{*}$ is a state, then

$$
\begin{aligned}
(i d \otimes \rho)\left(\hat{\Delta}^{o p}(x)\right) & =(i d \otimes \rho \otimes \omega)\left(\hat{\Delta}^{o p}(x) \otimes 1\right) \\
& =(i d \otimes \rho \otimes \omega)(\hat{\beta}(x \otimes 1)) \in N_{\hat{\beta}}\left(\left(\mathbb{G}_{\beta} \ltimes P\right)(N)\right) .
\end{aligned}
$$

It follows from Lemma 4.4 that $N$ is contained in $N_{\hat{\beta}}\left(\left(\mathbb{G}_{\beta} \ltimes P\right)(N)\right)$. Therefore, we have proven that $N=N_{\hat{\beta}}\left(\left(\mathbb{G}_{\beta} \ltimes P\right)(N)\right)$.

Let $L$ be as above. Take any state $\phi \in A_{*}$. Then, by Lemma 4.4 we have

$$
\begin{aligned}
N & =\left\{(i d \otimes \omega)\left(\hat{\Delta}^{o p}(x)\right): x \in N, \omega \in \widehat{M}_{*}\right\}^{\prime \prime} \\
& =\left\{(i d \otimes \omega \otimes \phi)\left(\hat{\Delta}^{o p}(x) \otimes 1\right): x \in N, \omega \in \widehat{M}_{*}\right\}^{\prime \prime} \\
& =\left\{(i d \otimes \omega \otimes \phi)(\hat{\beta}(x \otimes 1)): x \in N, \omega \in \widehat{M}_{*}\right\}^{\prime \prime} \subseteq N_{\hat{\beta}}(L) .
\end{aligned}
$$

In the meantime, $L$ is clearly included in $\left(\mathbb{G}_{\beta} \ltimes P\right)(N)$. Hence, by the result of the previous paragraph, we get $N_{\hat{\beta}}(L) \subseteq N_{\hat{\beta}}\left(\left(\mathbb{G}_{\beta} \ltimes P\right)(N)\right)=N$.

Lemma 4.7. Let $\mathbb{G}=(M, \Delta, \varphi, \psi)$ be a locally compact quantum group. Then $M^{\mathcal{G}(\mathbb{G})}=\mathbf{C}$ if and only if $\mathbb{G}$ is commutative.

Proof. If $\mathbb{G}$ is commutative, then we clearly have $M^{\mathcal{G}(\mathbb{G})}=\mathbf{C}$.

Put $N:=I G(\hat{\mathbb{G}})^{\prime \prime}$. It is easy to see that $N$ is a two-sided co-ideal (von Neumann subalgebra) of $\widehat{M}$ (more precisely, of $\widehat{\mathbb{G}}$ ). Moreover, we have $M^{\mathcal{G}(\mathbb{G})}=M \cap N^{\prime}$. From [4] Théorème 3.3] (which is still valid for a locally compact quantum group), it follows that $N=\widehat{M}$ if $M^{\mathcal{G}(\mathbb{G})}=\mathbf{C}$. Then $\widehat{\mathbb{G}}$ is cocommutative.

As explained in Section 1, the mapping $\left.v \in I G\left(\mathbb{G}^{\prime}\right) \longmapsto \operatorname{Ad} v\right|_{\widehat{M}} \in \mathcal{G}\left(\widehat{\mathbb{G}}^{o p}\right)$ is a topological isomorphism. Let $\beta_{v} \in \mathcal{G}\left(\widehat{\mathbb{G}}^{o p}\right)$ stand for the image of $v \in I G\left(\mathbb{G}^{\prime}\right)$ under this isomorphism.

Theorem 4.8. Let $\alpha$ be a minimal and integrable action of a locally compact quantum group $\mathbb{G}=(M, \Delta, \varphi, \psi)$ on a factor $A$. We set $P:=\mathcal{N}\left(A^{\alpha}\right)^{\prime \prime}$ and define $P_{1}$ to be the basic extension of $P \subseteq A$, i.e, $P_{1}:=J_{\omega} P^{\prime} J_{\omega}$. With the isomorphism $\rho: \mathbb{G}_{\alpha} \ltimes A \longrightarrow A_{1}$, put $Q_{1}:=\rho^{-1}\left(P_{1}\right)$. Let $w(\cdot): \mathcal{N}\left(A^{\alpha}\right) \longrightarrow I G(\mathbb{G})$ and $v(\cdot): \mathcal{N}\left(A^{\alpha}\right) \longrightarrow I G\left(\mathbb{G}^{\prime}\right)$ be the maps obtained in Proposition 4.2.

(1) The maps $w(\cdot), v(\cdot)$ are continuous homomorphisms with $\mathcal{U}\left(A^{\alpha}\right)$ their kernel. 
(2) We have

$$
\begin{aligned}
Q_{1}=\left(\mathbb{G}_{\alpha} \ltimes A\right)^{\left\{\theta_{u}: u \in \mathcal{N}\left(A^{\alpha}\right)\right\}} & =\left(\mathbb{G}_{\alpha} \ltimes A\right)^{\left\{\theta_{\beta_{v(u)}}: u \in \mathcal{N}\left(A^{\alpha}\right)\right\}} \\
& =\left(\mathbb{G}_{\alpha} \ltimes A\right)\left(\widehat{M}^{\left\{\beta_{v(u)}: u \in \mathcal{N}\left(A^{\alpha}\right)\right\}}\right) .
\end{aligned}
$$

(3) We have $N_{\hat{\alpha}}\left(Q_{1}\right)=\widehat{M}^{\left\{\beta_{v(u)}: u \in \mathcal{N}\left(A^{\alpha}\right)\right\}}$.

Proof. (1) This is straightforward.

(2) With the original definition of $\theta_{u}\left(u \in \mathcal{N}\left(A^{\alpha}\right)\right)$, we clearly have that $\left(A_{1}\right)^{\left\{\theta_{u}: u \in \mathcal{N}\left(A^{\alpha}\right)\right\}}=P_{1}$. So we get the first equality of our assertion. By Proposition 4.2 (2), we have $\theta_{u}=\beta_{v(u)}$, which yields the second equality. The last equality follows from the fact that $\theta_{\beta}$ always satisfies $(\beta \otimes i d) \circ \hat{\alpha}=\hat{\alpha} \circ \theta_{\beta}$ due to Proposition 3.1 .

(3) This follows from Part (2) and Lemma 4.6.

Let $P \subseteq Q$ be an inclusion of von Neumann algebras. If the normalizer group $\mathcal{N}(P)$ of $P$ in $Q$ generates $Q$, we say that $P$ is regular in $Q$.

The next theorem is a direct generalization of [20, Theorem 3.6], where we treated only the case where $\mathbb{G}$ is finite-dimensional.

Theorem 4.9. Suppose that $\alpha$ is a minimal and integrable action of a locally compact quantum group $\mathbb{G}=(M, \Delta, \varphi, \psi)$ on a factor $A$.

(1) If $A^{\alpha}$ is regular in $A$, then $\mathbb{G}$ is cocommutative.

(2) If $A^{\alpha}$ is infinite, or if $A$ is finite and $\mathbb{G}$ is finite-dimensional, then the cocommutativity of $\mathbb{G}$ implies the regularity of $A^{\alpha}$ in $A$.

Proof. Retain the notation employed in Theorem 4.8

(1) Suppose that $A^{\alpha}$ is regular in $A$. Then $Q_{1}=\alpha(A)$. By Theorem 4.8 (3), we get

$$
\widehat{M} \cap\left\{v(u): u \in \mathcal{N}\left(A^{\alpha}\right)\right\}^{\prime}=\widehat{M}^{\left\{\beta_{v(u)}: u \in \mathcal{N}\left(A^{\alpha}\right)\right\}}=\mathbf{C} .
$$

In particular, $\widehat{M} \cap\left\{w(u): u \in \mathcal{N}\left(A^{\alpha}\right)\right\}^{\prime}=\mathbf{C}$, because we have $J \widehat{M} J=\widehat{M}$ in general (see [10]). Since $\left\{w(u): u \in \mathcal{N}\left(A^{\alpha}\right)\right\}^{\prime \prime}$ is a two-sided co-ideal of $\mathbb{G}$, it follows from (2.1) that

$$
\left\{w(u): u \in \mathcal{N}\left(A^{\alpha}\right)\right\}^{\prime \prime}=M \cap\left(\widehat{M} \cap\left\{w(u): u \in \mathcal{N}\left(A^{\alpha}\right)\right\}^{\prime}\right)^{\prime}=M .
$$

Hence $\mathbb{G}$ is cocommutative.

(2) Assume that $A^{\alpha}$ is infinite, or that $A$ is finite and $\mathbb{G}$ is finite-dimensional. Let $v \in I G\left(\mathbb{G}^{\prime}\right)$. It follows from the proof of Lemma 3.5 that $\operatorname{Ad}(v \otimes 1)$ is in Aut $\left(\mathbb{G}_{\alpha} \ltimes A / \alpha(A)\right)$. By assumption, it also follows from Proposition 4.2 and the discussion preceding it that $u \in \mathcal{N}\left(A^{\alpha}\right) \longmapsto \theta_{u}=\operatorname{Ad}(v(u) \otimes 1) \in$ Aut $\left(\mathbb{G}_{\alpha} \ltimes A / \alpha(A)\right)$ is an isomorphism. Hence there is a unique $u \in \mathcal{N}\left(A^{\alpha}\right)$ such that

$$
\operatorname{Ad}(v \otimes 1)=\theta_{u}=\operatorname{Ad}(v(u) \otimes 1) .
$$

By the proof of Lemma3.5 again, we must have $v=v(u)$. Therefore, the map $v(\cdot)$ is surjective, i.e., $v\left(\mathcal{N}\left(A^{\alpha}\right)\right)=I G\left(\mathbb{G}^{\prime}\right)$. So, if $\mathbb{G}$ is cocommutative, then $v\left(\mathcal{N}\left(A^{\alpha}\right)\right)^{\prime}=$ $M$. From this, it follows that

$$
\widehat{M}^{\left\{\beta_{v(u)}: u \in \mathcal{N}\left(A^{\alpha}\right)\right\}}=\widehat{M} \cap M=\mathbf{C} .
$$

By Theorem $4.8(3), N_{\hat{\alpha}}\left(Q_{1}\right)=$ C. This means that $Q_{1}=\left(\mathbb{G}_{\alpha} \ltimes A\right)^{\hat{\alpha}}=\alpha(A)$. Therefore $A=P$. 
Next we would like to discuss Theorem 4.9 (2) in the case where $A$ is finite and $\mathbb{G}$ is infinite-dimensional.

Let $\Gamma$ be a (countable) discrete group and $\alpha$ be a minimal co-action of $\Gamma$ (i.e., a minimal action of $\widehat{\mathbb{G}}(\Gamma))$ on a type $\mathrm{II}_{1}$ factor $A$. For any $\gamma \in \Gamma$, define

$$
A^{\alpha}(\gamma):=\{a \in A: \alpha(a)=\lambda(\gamma) \otimes a\}
$$

and call it the eigensubspace of $\gamma$. The subspaces $\left\{A^{\alpha}(\gamma)\right\}_{\gamma \in \Gamma}$ played a vital part in defining the Connes spectrum $\Gamma(\alpha)$ of $\alpha$ in [18].

Proposition 4.10. Let $\Gamma, \alpha$ and $A$ be as above. For any $\gamma \in \Gamma$, the eigensubspace $A^{\alpha}(\gamma)$ contains a unitary.

Proof. By [18, Theorem 3.17], $A^{\alpha}(\gamma)^{*} A^{\alpha}(\gamma)$ is $\sigma$-weakly dense in $A^{\alpha}$, so that $A^{\alpha}(\gamma)$ contains plenty of nonzero elements for any $\gamma \in \Gamma$. Fix an arbitrary $\gamma \in \Gamma \backslash\{e\}$. Put

$$
B:=\left\{\left[\begin{array}{cc}
a & X \\
Y^{*} & b
\end{array}\right]: a, b \in A^{\alpha}, X, Y \in A^{\alpha}(\gamma)\right\} .
$$

By using the minimality of $\alpha$ and the fact that $A^{\alpha} \cap A^{\alpha}(\gamma)=\{0\}(\forall \gamma \neq e)$, one can easily verify that $B$ is a subfactor of $M_{2}(A)=A \otimes M_{2}(\mathbf{C})$. Accordingly, the unique tracial state on $A \otimes M_{2}(\mathbf{C})$ restricts to that of $B$. So the projections

$$
\left[\begin{array}{ll}
1 & 0 \\
0 & 0
\end{array}\right], \quad\left[\begin{array}{ll}
0 & 0 \\
0 & 1
\end{array}\right]
$$

are equivalent in $B$, since they have equal traces there. Hence there exists an isometry $Y \in A^{\alpha}(\gamma)$. Since $A$ is finite, $Y$ is a unitary.

Theorem 4.11. Suppose that $\alpha$ is a minimal, integrable action of a cocommutative locally compact quantum group $\mathbb{G}=(M, \Delta, \varphi, \psi)$ on a $\mathrm{II}_{1}$ factor $A$. Then $A^{\alpha}$ is regular in $A$.

Proof. Since $A$ is finite, it follows that $\mathbb{G}$ must be of compact type. Hence $\mathbb{G}$ has the form $\mathbb{G}=\widehat{\mathbb{G}}(\Gamma)$ for a unique (countable) discrete group $\Gamma$. By Proposition $4.10 \mathrm{ev-}$ ery eigensubspace $A^{\alpha}(\gamma)$ contains a unitary $V(\gamma)$. Clearly $V(\gamma)$ belongs to $\mathcal{N}\left(A^{\alpha}\right)$. So it remains to show that $\{V(\gamma)\}_{\gamma \in \Gamma}$ and $A^{\alpha}$ together generate $A$. But this follows from the next two facts: (i) $A$ is generated by $\left\{A^{\alpha}(\gamma)\right\}_{\gamma \in \Gamma}$; (ii) $A^{\alpha}(\gamma)$ has the form $A^{\alpha}(\gamma)=A^{\alpha} V(\gamma)$ for any $\gamma \in \Gamma$.

Remark. We remark that, for a minimal, integrable action $\alpha$ on an infinite factor $A$ with $A^{\alpha}$ finite, the cocommutativity of $\mathbb{G}$ does NOT in general imply the regularity of $A^{\alpha}$. In fact, suppose that $A$ is a factor of type $\mathrm{III}_{\lambda}(0<\lambda<1)$. Take a faithful normal state $\omega$ on $A$ with $\sigma_{T}^{\omega}=i d_{A}$, where $T:=-2 \pi / \log \lambda$. We regard this modular action as an action $\alpha$ of the one-dimensional torus $\mathbf{T}$ on $A$. It is well known that $\alpha$ is a minimal (integrable) action. Note that $A^{\alpha}$, the centralizer of $\omega$, is a factor of type $\mathrm{II}_{1}$. Let $u$ be in $\mathcal{N}\left(A^{\alpha}\right)$. It is easy to see that $u^{*} \alpha_{z}(u)$ lies in $\left(A^{\alpha}\right)^{\prime} \cap A=\mathbf{C}$. It follows that $u$ belongs to some spectral subspace $A^{\alpha}(n):=\left\{a \in A: \alpha_{z}(a)=z^{n} a(\forall z \in \mathbf{T})\right\}$ $(n \in \mathbf{Z})$ of the action $\alpha$. But, according to [14, Lemma 1.6], every spectral subspace $A^{\alpha}(n)$ except $A^{\alpha}(0)=A^{\alpha}$ contains no unitary. This shows that $\mathcal{N}\left(A^{\alpha}\right)$ is contained in $A^{\alpha}$. Therefore $A^{\alpha}$ is not regular in $A$. 


\section{Remarks on ILP's Galois CORRespondence}

Let $\alpha$ be an action of a compact Kac algebra $\mathbb{G}=(M, \Delta, \varphi, \psi)$ on a factor $A$. In what follows, the action $\alpha$ is assumed to be minimal and integrable, but we do not necessarily assume that $A^{\alpha}$ is infinite.

In [7], a complete Galois correspondence for a minimal action of a compact Kac algebra is obtained. According to [7. Theorem 4.4], the map $N \longmapsto A(N)$ gives a one-to-one correspondence between the lattice of right co-ideals of $\mathbb{G}$ and that of intermediate subfactors of $A^{\alpha} \subseteq A$.

Proposition 5.1. The inverse map of ILP's Galois correspondence cited above is given by $B \longmapsto N_{\alpha}(B)$.

Proof. Let $L, \bar{A}$ and $\bar{\alpha}$ be as in the proof of Theorem 3.6 . Then it is easy to check that, for any intermediate subfactor $B$ of $A^{\alpha} \subseteq A$ and any right co-ideal $N$ of $\mathbb{G}$, one has

$$
\bar{A}(N)=L \otimes A(N), \quad N_{\bar{\alpha}}(L \otimes B)=N_{\alpha}(B) .
$$

(Note that any intermediate subfactor $C$ of $\bar{A}^{\bar{\alpha}} \subseteq \bar{A}$ has the form $C=L \otimes B$ for a unique $B$ as above, thanks to [7, Theorem 3.9] and (the proof of) [15, Lemma 2.1].) Therefore, by considering $\bar{A}$ instead of $A$ itself if necessary, we may assume from the outset that $A^{\alpha}$ is infinite. Then $\alpha$ is dominant by [17, Theorem 2.2] or [16. Proposition 6.4]. So there exists an outer action $\beta$ of $\widehat{\mathbb{G}}^{\prime}$ on $A^{\alpha}$ such that $\{A, \alpha\}$ is conjugate to $\left\{\widehat{\mathbb{G}}^{\prime}{ }_{\beta} \ltimes A^{\alpha}, \hat{\beta}\right\}$. Hence we assume that $A=\widehat{\mathbb{G}}^{\prime} \beta \ltimes A^{\alpha}$ and $\alpha=\hat{\beta}$.

First, by Lemma 4.6 $N=N_{\alpha}(A(N))$ for any right co-ideal $N$ of $\mathbb{G}$.

Let $B$ be an intermediate subfactor of $A^{\alpha} \subseteq A$. Choose the unique right co-ideal $N_{1}$ such that $B=A\left(N_{1}\right)$ by using [7]. By the result of the preceding paragraph, we obtain $A\left(N_{\alpha}(B)\right)=A\left(N_{\alpha}\left(A\left(N_{1}\right)\right)\right)=A\left(N_{1}\right)=B$. This completes the proof.

The following proposition is regarded as an extension of [20, Theorem 3.5], where we discussed the case of $\mathbb{G}$ being finite-dimensional.

Proposition 5.2. Suppose that $\alpha$ is a minimal action of a compact Kac algebra $\mathbb{G}=(M, \Delta, \varphi, \psi)$ on a factor $A$ with $A^{\alpha}$ infinite. Then $N_{\alpha}\left(\mathcal{N}\left(A^{\alpha}\right)^{\prime \prime}\right)=I G(\mathbb{G})^{\prime \prime}$, i.e., the right co-ideal of $\mathbb{G}$ corresponding to the intermediate subfactor $\mathcal{N}\left(A^{\alpha}\right)^{\prime \prime}$ is $I G(\mathbb{G})^{\prime \prime}$.

Proof. Let $P_{1}$ be the basic extension of the inclusion $P:=\mathcal{N}\left(A^{\alpha}\right)^{\prime \prime} \subseteq A$. With $\rho$ the isomorphism introduced just after Lemma 4.1, set $Q_{1}:=\rho^{-1}\left(P_{1}\right)$. By Lemma 4.1 and Theorem 4.8, we have $\widehat{M}^{\mathcal{G}\left(\widehat{\mathbb{G}}^{o p}\right)}=N_{\hat{\alpha}}\left(Q_{1}\right)$. From this and Corollary 6.3, we obtain $\widehat{M}^{\mathcal{G}}\left(\widehat{\mathbb{G}}^{o p}\right)=\hat{R}\left(\widehat{M} \cap N_{\alpha}(P)^{\prime}\right)$. In other words, $\widehat{M} \cap I G\left(\mathbb{G}^{\prime}\right)^{\prime}=\hat{R}\left(\widehat{M} \cap N_{\alpha}(P)^{\prime}\right)$. Hence, by 2.1, we have

$$
\begin{aligned}
N_{\alpha}(P) & =M \cap\left(\widehat{M} \cap N_{\alpha}(P)^{\prime}\right)^{\prime}=M \cap \hat{R}\left(\widehat{M} \cap I G\left(\mathbb{G}^{\prime}\right)^{\prime}\right)^{\prime} \\
& =M \cap\left(\widehat{M} \cap J I G\left(\mathbb{G}^{\prime}\right)^{\prime} J\right)^{\prime}=M \cap\left(\widehat{M} \cap I G(\mathbb{G})^{\prime}\right)^{\prime}=I G(\mathbb{G})^{\prime \prime} .
\end{aligned}
$$

Thus we are done.

Remark. The above proposition may be used in order to prove Theorem 4.9 in the case of compact Kac algebra actions. 


\section{ApPENDix}

Let $\alpha$ be a minimal and integrable action of a locally compact quantum group $\mathbb{G}=(M, \Delta, \varphi, \psi)$ on a factor $A$. We fix a faithful normal semifinite weight $\omega$ on $A$ and regard $A$ as acting on $H_{\omega}$. Denote by $\tilde{J}_{\omega}$ the modular conjugation of the dual weight $\tilde{\omega}$. The canonical implementation $U$ of $\alpha$ associated to $\omega$ is then given by $U=\tilde{J}_{\omega}\left(\hat{J} \otimes J_{\omega}\right)$. The basic extension of $A^{\alpha} \subseteq A$ is denoted by $A_{1}:=J_{\omega}\left(A^{\alpha}\right)^{\prime} J_{\omega}$.

Let $\rho$ be the isomorphism $\rho: \mathbb{G}_{\alpha} \ltimes A \longrightarrow A_{1}$ that appeared in Section 3. Identity (4.2) can be rewritten in the form:

$$
\rho(\lambda(\phi) \otimes 1)=(\phi \otimes i d)\left(U^{*}\right) \quad\left(\phi \in M_{*}\right) .
$$

Let $A_{2}$ be the basic extension of $A \subseteq A_{1}$. Since $B\left(H_{\varphi}\right) \otimes A$ is the basic extension of $\alpha(A) \subseteq \mathbb{G}_{\alpha} \ltimes A$, the above isomorphism $\rho$ can be extended to the isomorphism, still denoted by $\rho$, from $B\left(H_{\varphi}\right) \otimes A$ onto $A_{2}$. Since $B\left(H_{\varphi}\right) \otimes A \cap\left(\mathbf{C} \otimes A^{\alpha}\right)^{\prime}=B\left(H_{\varphi}\right) \otimes \mathbf{C}$, the equation

$$
\Pi(T):=\rho(T \otimes 1) \quad\left(T \in B\left(H_{\varphi}\right)\right)
$$

defines a $*$-isomorphism $\Pi$ from $B\left(H_{\varphi}\right)$ onto $A_{2} \cap\left(A^{\alpha}\right)^{\prime}$. Since $B\left(H_{\varphi}\right) \otimes A \cap$ $\alpha(A)^{\prime}=M^{\prime} \otimes \mathbf{C}$ and $\mathbb{G}_{\alpha} \ltimes A \cap\left(\mathbf{C} \otimes A^{\alpha}\right)^{\prime}=\widehat{M} \otimes \mathbf{C}$, it follows from (6.1) (recall that $\left.\widehat{M}=\left\{\lambda(\phi): \phi \in M_{*}\right\}^{\prime \prime}\right)$ that

$$
A_{2} \cap A^{\prime}=\Pi\left(M^{\prime}\right), \quad A_{1} \cap\left(A^{\alpha}\right)^{\prime}=\Pi(\widehat{M})=\left\{(\phi \otimes i d)\left(U^{*}\right): \phi \in M_{*}\right\}^{\prime \prime} .
$$

Since $\hat{R}(\lambda(\phi))=J \lambda(\phi)^{*} J=\lambda(\phi \circ R)$, it follows from (6.1) that

$$
\Pi(\hat{R}(z))=J_{\omega} \Pi(z)^{*} J_{\omega} \quad(z \in \widehat{M}) .
$$

Lemma 6.1 (Proposition 4.4, [4]). For any intermediate subfactor $B$ of $A^{\alpha} \subseteq A$, we have

$$
N_{\alpha}(B)^{\prime} \otimes \mathbf{C}=B\left(H_{\varphi}\right) \otimes A \cap \alpha(B)^{\prime} .
$$

Equivalently, $\Pi\left(N_{\alpha}(B)^{\prime}\right)=A_{2} \cap B^{\prime}$.

Proof. Since $B\left(H_{\varphi}\right) \otimes A \cap\left(\mathbf{C} \otimes A^{\alpha}\right)^{\prime}=B\left(H_{\varphi}\right) \otimes \mathbf{C}$, we see that $B\left(H_{\varphi}\right) \otimes A \cap \alpha(B)^{\prime}=$ $\left\{T \otimes 1: T \in B\left(H_{\varphi}\right), T \otimes 1 \in \alpha(B)^{\prime}\right\}$. For $T \in B\left(H_{\varphi}\right)$, we have

$$
\begin{aligned}
& T \otimes 1 \in \alpha(B)^{\prime} \Longleftrightarrow \quad(\chi \otimes \phi)((T \otimes 1) \alpha(b))=(\chi \otimes \phi)(\alpha(b)(T \otimes 1)) \\
&\left(\chi \in B\left(H_{\varphi}\right)_{*}, \phi \in A_{*}\right) \\
& \Longleftrightarrow \quad \chi(T(i d \otimes \phi)(\alpha(b)))=\chi((i d \otimes \phi)(\alpha(b)) T) \\
& \quad\left(\chi \in B\left(H_{\varphi}\right)_{*}, \phi \in A_{*}\right) .
\end{aligned}
$$

The last condition is equivalent to $T$ being in $N_{\alpha}(B)^{\prime}$.

Lemma 6.2 (Corollaire 4.5, [4]). Let $B$ be an intermediate subfactor of $A^{\alpha} \subseteq A$.

(1) We have $\widehat{M} \cap N_{\alpha}(B)^{\prime} \otimes \mathbf{C}=\mathbb{G}_{\alpha} \ltimes A \cap \alpha(B)^{\prime}$, i.e., $\Pi\left(\widehat{M} \cap N_{\alpha}(B)^{\prime}\right)=A_{1} \cap B^{\prime}$.

(2) We have $N_{\alpha}(B)^{\prime} \otimes \mathbf{C}=\left(M^{\prime} \otimes \mathbf{C}\right) \vee\left(\mathbb{G}_{\alpha} \ltimes A \cap \alpha(B)^{\prime}\right)$, i.e., $A_{2} \cap B^{\prime}=$ $\left(A_{2} \cap A^{\prime}\right) \vee\left(A_{1} \cap B^{\prime}\right)$.

(3) Let $B_{1}:=J_{\omega} B^{\prime} J_{\omega}$ be the basic extension of $B \subseteq A$, and set $\tilde{B}_{1}:=\rho^{-1}\left(B_{1}\right)$. Then $\Pi\left(\hat{R}\left(\widehat{M} \cap N_{\alpha}(B)^{\prime}\right)\right)=B_{1} \cap\left(A^{\alpha}\right)^{\prime}$. In particular, $\tilde{B}_{1} \cap\left(\mathbf{C} \otimes A^{\alpha}\right)^{\prime}=$ $\hat{R}\left(\widehat{M} \cap N_{\alpha}(B)^{\prime}\right) \otimes \mathbf{C}$. 
Proof. (1) This follows from Lemma 6.1 and the identity $\mathbb{G}_{\alpha} \ltimes A \cap\left(\mathbf{C} \otimes A^{\alpha}\right)^{\prime}=$ $\widehat{M} \otimes \mathbf{C}$.

(2) This is due to the fact that $N_{\alpha}(B)=M \cap\left(\widehat{M} \cap N_{\alpha}(B)^{\prime}\right)^{\prime}$.

(3) This follows from Part (1) and (6.3).

Theorem 6.3. Let $B, B_{1}, \tilde{B}_{1}$ be as in Lemma 6.2. If $\mathbb{G}$ is a compact Kac algebra, then we have $B_{1}=A \vee\left(B_{1} \cap\left(A^{\alpha}\right)^{\prime}\right)$ and $\tilde{B}_{1}=\alpha(A) \vee \hat{R}\left(\widehat{M} \cap N_{\alpha}(B)^{\prime}\right) \otimes \mathbf{C}$. Moreover, $N_{\hat{\alpha}}\left(\tilde{B}_{1}\right)=\hat{R}\left(\widehat{M} \cap N_{\alpha}(B)^{\prime}\right)$.

Proof. For $\omega$, choose a faithful normal state $\omega_{0}$ on $A^{\alpha}$ and put $\omega:=\omega_{0} \circ T_{\alpha}$. By 7 . Theorem 3.9], there exists a (unique) conditional expectation $E_{B}$ from $A$ onto $B$. Let $e_{B}$ be the Jones projection of $B: e_{B} \Lambda_{\omega}(a)=\Lambda_{\omega}\left(E_{B}(a)\right)$. So $B_{1}=\left(A \cup\left\{e_{B}\right\}\right)^{\prime \prime}$. Since $e_{B} \in B_{1} \cap\left(A^{\alpha}\right)^{\prime}$, we find that $B_{1}=A \vee\left(B_{1} \cap\left(A^{\alpha}\right)^{\prime}\right)$. From Lemma 6.2 (3), it follows that $\tilde{B}_{1}=\alpha(A) \vee \hat{R}\left(\widehat{M} \cap N_{\alpha}(B)^{\prime}\right) \otimes \mathbf{C}$. From Lemma 4.6, we find that $N_{\hat{\alpha}}\left(\tilde{B}_{1}\right)=\hat{R}\left(\widehat{M} \cap N_{\alpha}(B)^{\prime}\right)$.

\section{REFERENCES}

[1] H. Araki, R. Haag, D. Kastler and M. Takesaki, Extension of KMS states and chemical potential, Commun. Math. Phys., 53 (1977), 97-134. MR 56:2042

[2] J. De Cannière, On the intrinsic group of a Kac algebra, Proc. London Math. Soc., 40 (1980), 1-20. MR 83d:46079

[3] J. Dixmier and O. Maréchal, Vecteurs totalisateurs d'une algèbre de von Neumann, Commun. Math. Phys., 22 (1971), 44-50. MR 45:5767

[4] M. Enock, Sous-facteurs intermédiaires et groupes quantiques mesurés, J. Operator Theory, 42 (1999), 305-330. MR 2000i:46057

[5] M. Enock and J.-M. Schwartz, Systèmes dynamiques généralisés et correspondances, J. Operator Theory, 11 (1984), 273-303. MR 86g:46097

[6] M. Enock and J.-M. Schwartz, Kac Algebras and Duality of Locally Compact Groups, Springer-Verlag, Berlin (1992). MR 94e:46001

[7] M. Izumi, R. Longo and S. Popa, A Galois correspondence for compact groups of automorphisms of von Neumann algebras with a generalization to Kac algebras, J. Funct. Anal., 155 (1998), 25-63. MR 2000c:46117

[8] H. Kosaki, Extension of Jones' theory on index to arbitrary factors, J. Funct. Anal., 66 (1986), 123-140. MR 87g:46093

[9] J. Kustermans and S. Vaes, Locally compact quantum groups, Ann. Sci. École Norm. Sup., $4^{e}$ série, t. 33 (2000), 837-934. MR 2002f:46108

[10] J. Kustermans and S. Vaes, Locally compact quantum groups in the von Neumann algebra setting, To appear in Math. Scand..

[11] M. Pimsner and S. Popa, Entropy and index for subfactors, Ann. Sci. École Norm. Sup., $4^{e}$ série, t. 19 (1986), 57-106. MR 87 m:46120

[12] J.-M. Schwartz, Sur la structure des algèbres de Kac, I, J. Funct. Anal., 34 (1979), 370-406. MR 83a:46072a

[13] Ş. Strătilă, Modular theory in operator algebras, Abacus Press, Tunbridge Wells, 1981. MR 85g:46072

[14] M. Takesaki, The structure of a von Neumann algebra with a homogeneous periodic state, Acta Math., 131 (1973), 79-121. MR 55:11067

[15] T. Teruya and Y. Watatani, Lattices of intermediate subfactors for type III factors, Arch. Math., 68 (1997), 454-463. MR 98d:46071

[16] S. Vaes, The unitary implementation of a locally compact quantum group action, J. Funct. Anal., 180 (2001), 426-480. MR 2002a:46100

[17] T. Yamanouchi, On dominancy of minimal actions of compact Kac algebras and certain automorphisms in $\operatorname{Aut}\left(\mathcal{A} / \mathcal{A}^{\alpha}\right)$, Math. Scand., 84 (1999), 297-319. MR 2000g:46101

[18] T. Yamanouchi, The Connes spectrum for actions of compact Kac algebras and factoriality of their crossed products, Hokkaido Math. J., 28 (1999), 409-434. MR 2000g:46100 
[19] T. Yamanouchi, Uniqueness of Haar measures for a quasi Woronowicz algebra, Hokkaido Math. J., 30 (2001), 105-112. MR 2002e:46075

[20] T. Yamanouchi, Description of the automorphism group Aut $\left(\mathcal{A} / \mathcal{A}^{\alpha}\right)$ for a minimal action of a compact Kac algebra and its application, J. Funct. Anal., 194 (2002), 1-16.

Department of Mathematics, Faculty of Science, Hokkaido University, Sapporo 0600810 JAPAN

E-mail address: yamanouc@math.sci.hokudai.ac.jp 